\title{
Phenotypic and genotypic characterization of levofloxacin- and moxifloxacin-resistant Mycobacterium tuberculosis clinical isolates in southern China
}

\author{
H. M. Adnan Hameed ${ }^{1,2 \#}$, Yaoju Tan ${ }^{3 \#}$, Md Mahmudul Islam ${ }^{1,2}$, Lingmin Guo ${ }^{1,2}$, Chiranjibi Chhotaray ${ }^{1,2}$, \\ Shuai Wang ${ }^{1,2}$, Zhiyong Liu ${ }^{1}$, Yamin Gao ${ }^{1,2}$, Shouyong Tan ${ }^{3}$, Wing Wai Yew ${ }^{4}$, Nanshan Zhong ${ }^{5}$, \\ Jianxiong Liu ${ }^{3}$, Tianyu Zhang ${ }^{1,2,5}$
}

${ }^{1}$ State Key Laboratory of Respiratory Disease, Guangzhou Institutes of Biomedicine and Health (GIBH), Chinese Academy of Sciences (CAS), Guangzhou 510530, China; ${ }^{2}$ University of Chinese Academy of Sciences (UCAS), Beijing 100049, China; ${ }^{3}$ State Key Laboratory of Respiratory Disease, Guangzhou Chest Hospital, Guangzhou 510095, China; ${ }^{4}$ Stanley Ho Centre for Emerging Infectious Diseases, The Chinese University of Hong Kong, Hong Kong, China; 'State Key Laboratory of Respiratory Disease, National Clinical Research Center for Respiratory Disease, The First Affiliated Hospital of Guangzhou Medical University, Guangzhou 510182, China

Contributions: (I) Conception and design: HMA Hameed, Y Tan, J Liu, N Zhong, T Zhang; (II) Administrative support: HMA Hameed, MM Islam, C Chhotaray, S Wang, T Zhang; (III) Provision of study materials or patients: T Zhang, Y Tan, S Tan, J Liu; (IV) Collection and assembly of data: HMA Hameed, L Guo, Z Liu, Y Gao; (V) Data analysis and interpretation: HMA Hameed, WW Yew, N Zhong, Y Tan, T Zhang; (VI) Manuscript writing: All authors; (VII) Final approval of manuscript: All authors.

\#These authors contributed equally to this work.

Correspondence to: Tianyu Zhang, PhD, PI. Guangzhou Institutes of Biomedicine and Health (GIBH), Chinese Academy of Sciences (CAS), Room A132, 190 Kaiyuan Ave, Science Park, Huangpu District, Guangzhou 510530, China. Email: zhang_tianyu@gibh.ac.cn; Jianxiong Liu. Guangzhou Chest Hospital, 62 Hengzhigang Road, Yuexiu District, Guangzhou 510095, China. Email: ljxer64@qq.com.

Background: Levofloxacin (LVX) and Moxifloxacin (MXF) are the cornerstones for treatment of multidrug-resistant tuberculosis (MDR-TB). China is one of the highest MDR- and fluoroquinolones (FQ)resistant TB burdens countries. DNA gyrase encoded by gyr genes is the main target of FQ in Mycobacterium tuberculosis (MTB). The prevalence and molecular characterization of LVX- and MXF-resistant MTB strains from southern China were examined in this study.

Methods: Drug susceptibility testing (DST) of 400 MTB clinical isolates was evaluated by proportion method on Löwenstein-Jensen (LJ) medium against ten drugs. The sequencing of entire gyr $A$ and gyrB genes and multiplex PCR were performed to distinguish the prevalence of mutant types in Beijing and non-Beijing genotypes.

Results: Three hundred and twenty-one out of four hundred (80.25\%) drug-resistant isolates (resistant $>$ one drug) were categorized as 83/321 (25.80\%) MDR, 174/321 (54.20\%) pre-XDR and 64/321 (19.93\%) XDRMTB. Overall, 303/400 (75.75\%) LVX- and 292/400 (73.00\%) MXF-resistant (R) MTB strains were identified. Two hundred seventy-one out of three hundred and three $(89.43 \%)$ resistant strains carried mutations in gyr $A$ and $91 / 303(30.03 \%)$ in gyrB. Interestingly, 18 novel mutations were detected in gyr $A$ and gyr $B$ genes. Mutations at (A90, D94) and (T500, G510, G512) frequently existed in QRDR(s) of gyrA and gyrB respectively in $286 / 400(71.50 \%)$ LVX $^{\mathrm{R}} \mathrm{MXF}^{\mathrm{R}}$ strains. The novel mutations in- and out-side the QRDR of gyrA (L105R, A126E, M127K, D151T, V165A) and gyrB (D461H, N499S, G520A) increased the sensitivity and consistency of genotypic tests. Notably, $25 \mathrm{LVX}^{\mathrm{R}} \mathrm{MXF}^{\mathrm{R}}$ strains were found with unknown resistance mechanisms.

Conclusions: Mutations in QRDR(s) were concomitantly associated with Beijing and non-Beijing genotypes. The prevalence of resistance and cross-resistance between LVX and MXF in MTB isolates from southern China was immensely higher than other countries. Our valuable findings provide the substantial implications to improve the reliability of genotypic diagnostic tests relying on potential resistance conferring mutations in entire gyr genes. 
Keywords: Mycobacterium tuberculosis (MTB); levofloxacin (LVX); moxifloxacin (MXF); cross-resistance; susceptibility testing; novel mutations

Submitted May 02, 2019. Accepted for publication Oct 21, 2019.

doi: $10.21037 /$ jtd.2019.11.03

View this article at: http://dx.doi.org/10.21037/jtd.2019.11.03

\section{Introduction}

Tuberculosis (TB) is a ninth major cause of morbidity and mortality around the globe that is ranked higher than HIV. Among the 10 million TB cases in 2017, an estimated 558,000 people developed resistance to rifampicin (the most effective first-line drug), of which $82 \%$ had multidrugresistant tuberculosis (MDR-TB) (1). Fluoroquinolones (FQ), the broad-spectrum antimicrobial agents with bactericidal activity against Mycobacterium tuberculosis (MTB) are used as second-line drugs (2). Currently, the third- and fourth-generation FQ [levofloxacin (LVX) and moxifloxacin (MXF)] showing high in vitro and in vivo activities are extensively used for the treatment of MDR-TB (defined as resistant to at least isoniazid and rifampicin) (2-4).

China has around $22 \%$ contribution to the global burden of MDR-TB and the highest prevalence of FQ-resistant MTB (5). This serious epidemic of drug-resistant TB is associated with poor treatment outcome in MDR-TB and extensive practice of FQ for the treatment of undiagnosed respiratory bacterial infections $(5,6)$, which further cause the emergence of pre-extensively drug-resistant (pre-XDR) and extensively drug-resistant (XDR) TB. Pre-XDR-TB is defined as MDR-TB associated with resistance to FQ or a second-line injectable (e.g., kanamycin, amikacin, or capreomycin), but not both while XDR-TB is defined as MDR-TB with additional resistance to any FQ and a second-line injectable drug (7). The earlier reports from different regions of China showed the various proportions of XDR among MDR-TB; $6.28 \%$ in Beijing (8), $12.6 \%$ in Shanghai (9), $12.8 \%$ in Xinjiang (10), 20\% in Shandong province (11).

In line with older FQ agents, the two new agents (LVX and MXF) inhibit DNA gyrase (a type II topoisomerase composed of $\alpha$ and $\beta$ subunits), restricting the cell's capacity for DNA replication and transcription $(12,13)$. Mutations in $g y r A$ and $\operatorname{gyr} B$ genes, particularly in the quinolone resistance-determining region (QRDR) of gyr $A$ (codons 74 to 113 ) and $\operatorname{gyr} B$ (codons 500 to 540 ), are the main reason of FQ resistance in MTB (14). The substitutions in these two genes alter the structure of the quinolone-binding pocket $(\mathrm{QBP})$ and may widely cause the cross-resistance among FQ. GyrA mainly involves in breaking and reuniting of DNA, whereas GyrB plays a role in ATPase activity (15). Generally, gyrA is considered as the most promising target of FQ and most of potential inhibitors for TB are developed against this target.

Numerous molecular-based diagnostic methods to detect gyrA and gyrB mutations in QRDR have been developed, but the sensitivity for predicting the prevalence of phenotypic FQ resistance is highly inconsistent, ranging from $80.5 \%$ in Shanghai (16), $89.7 \%$ in Beijing (17), $87.5 \%$ in France (18), 90.6\% in Germany (19) and 75.6\% was in Vietnam (20). Most commonly observed resistance conferring mutations in gyrA are A90V, S91P, and D94 (A, $\mathrm{N}, \mathrm{G}, \mathrm{H}, \mathrm{Y}$ ) while the mutation $\mathrm{G} 88 \mathrm{C}$ is rarely detected $(4,21)$. Similarly, R485 (H, C), S486F, D495N, T500 (A, H, N), G509A, N533T, N538 (T, D), T539P, E540 (V, D) amino acid substitutions have been frequently noticed in the gyrB gene (22). However, $\sim 60 \%$ of FQ-resistant MTB isolates without known mutations in QRDR of gyrA and gyr $B$ compromises the sensitivity and specificity $(2,12)$. Although LVX and MXF are used in treating XDR-TB patients (23), but their roles are not fully certain due to bacillary baseline and cross-resistance against the FQ (24).

In addition, most of studies infrequently report the cumulative variances in the molecular-based test performance and cross-resistance conflicting the sensitivity and specificity across the new generation FQ agents (25-27). To improve the accuracy of molecular diagnosis for precise treatment of TB patients, there is an urgent need of detailed analyses assessing the entire open reading frames of the gyr genes for clinically relevant readout to accumulate the persuasive evidence that various mutations interaction in/ out-side the QRDR can significantly affect the independent or cross-resistance between LVX and MXF in MTB clinical strains. The current study aimed to investigate the prevalence of genetic mutations in entire gyr genes and their association with independent or cross-resistance to LVX and MXF in MTB clinical strains from southern China. 


\section{Methods}

\section{Ethical Statement}

The current study was conducted in accordance with WHO guidelines and approved by the Ethics Committee of Guangzhou Chest Hospital (GZXK-2016-015).

\section{Collection of MTB clinical isolates}

In this study, 400 MTB clinical isolates (resistant to $\geq 1$ or more drug) were collected during 2016-2018 from TB patients at Guangzhou Chest Hospital, The Central Hospital of Southern China for TB. The demographic data were obtained from the electronical medical record and the species were identified using a commercial MPB64 monoclonal antibody assay (Genesis, Hangzhou, China) (28). MTB wild-type reference strain (H37Rv ATCC27294 319 ) was used as a control in this study.

\section{Drug susceptibility testing (DST)}

The DST of 400 MTB clinical strains was performed in line with WHO guidelines using a proportion method on Löwenstein-Jensen (LJ) medium against 10 anti-TB drugs with the following critical concentrations $(\mu \mathrm{g} / \mathrm{mL})$; isoniazid 0.2 , rifampicin 40.0 , streptomycin 4.0 , ethambutol 2.0, MXF 1.0, LVX 2.0, amikacin 30.0, rifabutin 20.0, prothionamide 40.0 and para-aminosalicylic acid 1.0 (29-32). The serially diluted bacterial suspension of MTB strains were inoculated onto $\mathrm{LJ}$ slants with and without drugs and incubated at $37{ }^{\circ} \mathrm{C}$ for 42 days to read up the results. A strain was considered resistant to a tested drug when MTB growth rate is $\geq 1 \%$ compared with the drugfree control $(29,32)$.

\section{gyrA and gyrB genes amplification and sequencing analysis}

DNA extracts were prepared from pure and freshly grown MTB colonies as previously described (33). A loopful of MTB colonies was suspended into $500 \mu \mathrm{L}$ of Tris-EDTA buffer (10 mM Tris-HCl, $1 \mathrm{mM}$ EDTA, $\mathrm{pH}$ 8.0) and heated up to $80^{\circ} \mathrm{C}$ for 20 minutes. Finally, the crude DNA was used as a template for PCR amplification. The entire open reading frames of gyrA (product size $=2,849 \mathrm{bp}$ ) and $\operatorname{gyr} B$ (product size $=2,336 \mathrm{bp}$ ) including their ( $\geq 170 \mathrm{bp}$ ) 5'upstream region to $(\geq 170 \mathrm{bp}) 3$ 'downstream region were amplified in 400 MTB clinical strains using the newly designed primers in this study (gyrAF: 5'-TGCGTCAAGTGACGCTGGAC-3',
gyrAR: 5'-ACGGTCGACCAAGCCATCCG-3'), (gyrBF: 5'-TATGCCGGACGTCGGGACGC-3', gyrBR: 5'-TCATCGCATAGTCGATGTAG-3'). PCR products were observed on $0.7 \%$ to $1.0 \%$ agarose gels, purified by PCR purification kit (Qiagen, Hilden, Germany) and sent to BGI (Guangzhou, China) for Sanger sequencing. The sequencing data were aligned with the standard MTB H37Rv reference sequence (GenBank accession number NC_000962) using CLC Sequence Viewer; version 7.7.1 (Qiagen).

\section{PCR based detection of Beijing and non-Beijing genotypes}

PCR based detection method was used to differentiate Beijing genotype and non-Beijing genotype MTB strains as previously described $(34,35)$, a region spanning genes $R v 2816$ to $R v 2819$ and part of $R v 2820$ is missing in all Beijing genotype of MTB strains. So, the primer set of BJ-F: 5'-ACCGAGCTGATCAAACCCG-3' and BJR: 5'-ATGGCACGGCCGACCTGAATGAACC-3' was used to amplify 239-bp fragment containing region specific part of $R v 2819$ and part of $R v 2820$. Whereas, Non-Beijing genotype MTB strains were detected by amplification of 539-bp PCR fragment using primer set of NBJ-F: 5'-GATCGCTTGTTCTCAGTGCAG-3' and NBJ-R: 5'-CGAAGGAGTACCACGTGGAG-3' covering the region specific for $R v 2819$. The PCR products were observed on $2 \%$ agarose gels.

\section{Statistical analysis}

Phenotypic drug susceptibility testing was used as the reference for evaluation. Genotypic LVX and MXF resistance was defined as the prevalence of resistance conferring nonsynonymous mutations in- and out-side the QRDR of gyr $A$ and gyrB genes. The data was processed statistically to measure the sensitivity, specificity, accuracy, odd ratio and $95 \%$ confidence interval (CI) by using MEDCALC ${ }^{\circledR}$ statistical software (URL: https://www.medcalc.org/calc/ diagnostic_test.php; https://www.medcalc.org/calc/odds_ ratio.php). $\mathrm{P}<0.05$ was considered statistically significant.

\section{Results}

\section{Demographic characteristics}

Of the 400 studied MTB clinical isolates, 281 (70.25\%) were from male patients and 119 (29.75\%) were from female patients. Age-wise analysis exhibited that MTB isolates 
Table 1 Demographic characteristics of 400 MTB clinical isolates

\begin{tabular}{|c|c|c|c|c|c|c|c|c|}
\hline \multirow{2}{*}{ Characteristics } & \multicolumn{2}{|c|}{ No. of clinical isolates (\%) } & \multirow{2}{*}{ OR $(95 \% \mathrm{Cl})$} & \multirow{2}{*}{$P$ value } & \multicolumn{2}{|c|}{ No. of clinical isolates (\%) } & \multirow{2}{*}{ OR $(95 \% \mathrm{Cl})$} & \multirow{2}{*}{$P$ value } \\
\hline & $\operatorname{LVX}^{R}(n=303)$ & $\operatorname{LVX}^{S}(n=97)$ & & & $\operatorname{MXF}^{R}(n=292)$ & $\operatorname{MXF}^{S}(n=108)$ & & \\
\hline \multicolumn{9}{|l|}{ Gender } \\
\hline Female & $92(30.36)$ & $27(27.83)$ & 1.00 (Ref.) & - & $95(32.53)$ & $29(26.85)$ & 1.00 (Ref.) & - \\
\hline \multicolumn{9}{|l|}{ Age group } \\
\hline $20-40$ & $79(26.07)$ & $41(42.26)$ & 1.00 (Ref.) & - & $83(28.42)$ & $49(45.37)$ & 1.00 (Ref.) & - \\
\hline $40-60$ & $121(39.93)$ & $34(35.05)$ & $1.84(1.08-3.15)$ & 0.02 & $118(40.41)$ & $30(27.77)$ & $2.32(1.36-3.96)$ & 0.0020 \\
\hline$>60$ & $91(30.03)$ & $9(9.27)$ & $5.24(2.40-11.46)$ & $<0.0001$ & $84(28.76)$ & $7(6.48)$ & $7.08(3.03-16.54)$ & $<0.0001$ \\
\hline \multicolumn{9}{|l|}{ Treatment history } \\
\hline New case & 94 (31.02) & $43(44.32)$ & 1.00 (Ref.) & - & $99(33.90)$ & $51(47.22)$ & 1.00 (Ref.) & - \\
\hline
\end{tabular}

$\mathrm{P}<0.05$.
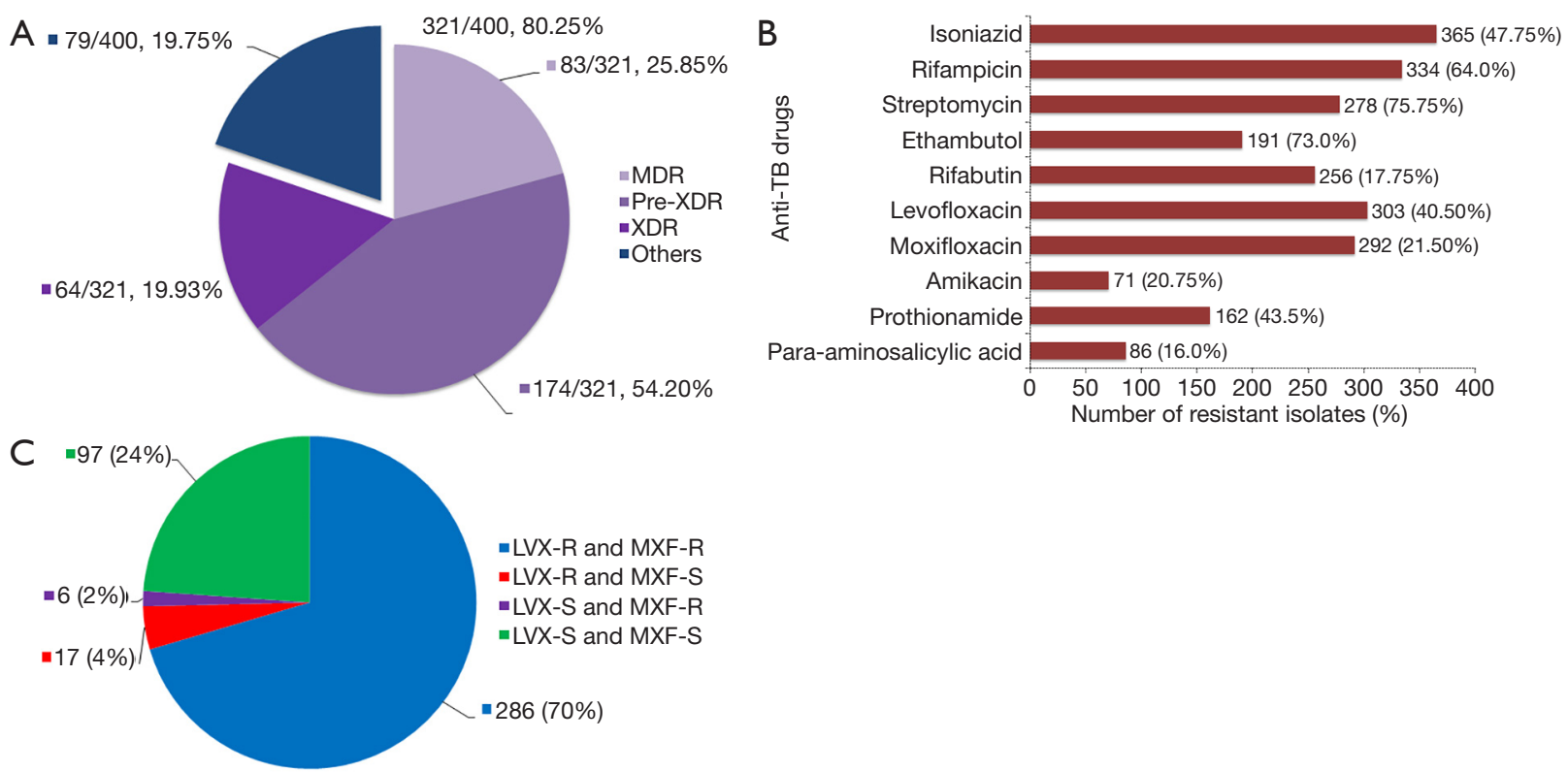

Figure 1 (A) Prevalence of MDR-, pre-XDR- and XDR-TB strains in 400 Mycobacterium tuberculosis clinical isolates; (B) drug susceptibility testing against ten anti-tuberculosis drugs; (C) LVX and MXF resistance pattern in Mycobacterium tuberculosis clinical isolates.

belonged to patients age range of $18-86$ years with a mean age of 42.7 years. In addition, majority of TB patients $263 / 400(65.75 \%)$ listed in this study were retreated whereas $137 / 400$ (34.25\%) were registered as new TB cases. The detailed demographic characteristics are explained in Table 1.

\section{Drug susceptibility testing outcome}

DST of 400 MTB clinical isolates categorized the $321 / 400$
$(80.25 \%)$ drug-resistant isolates (which have resistance against more than one drug) as $83 / 321(25.80 \%)$ MDR, 174/321 (54.20\%) pre-XDR and 64/321 (19.93\%) XDRMTB strains. While the remaining 79/400 (19.75\%) MTB isolates had random or distinct pattern of drug resistance (mono- and poly-resistant strains) (Figure 1A). Of the total isolates, $303 / 400(75.75 \%) \mathrm{LVX}^{\mathrm{R}}$, and $292 / 400$ $(73.00 \%) \mathrm{MXF}^{\mathrm{R}} \mathrm{MTB}$ isolates were identified in this study (Figure 1B). These isolates were observed with the 
pattern of 286/400 $\mathrm{LVX}^{\mathrm{R}} \mathrm{MXF}^{\mathrm{R}}, 17 / 400 \mathrm{LVX}^{\mathrm{R}} \mathrm{MXF}^{\mathrm{S}}, 6 / 400$ $\mathrm{LVX}^{\mathrm{S}} \mathrm{MXF}^{\mathrm{R}}$ and $97 / 400 \mathrm{LVX}^{\mathrm{S}} \mathrm{MXF}^{\mathrm{S}}$ MTB strains (Figure 1C). Overall, in this study MTB isolates were resistant to at least one or more drugs as there was no any isolate was included which was fully susceptible to all tested drugs.

\section{Association of gyrA and gyrB mutations with LVX-and MXF-resistance pattern}

Molecular diagnosis was performed for both susceptible and resistant MTB clinical isolates. The mutations identified in gyrA and gyrB genes are summarized in Table 2. Among the $286 / 400 \mathrm{LVX}^{\mathrm{R}} \mathrm{MXF}^{\mathrm{R}}$ strains, 254/286 (88.81\%) strains carried missense mutations in entire gyr $A$ gene including 5 novel mutations and 237/254 (93.30\%) of them were found with mutations only in QRDR of gyrA. The mutations A90V, D94N, D94G and D94A were frequently detected in gyrA QRDR. However, the new mutations (A126E, M127K) in MTB strains without QRDR mutations were found to be involved in resistance against both LVX and MXF.

Similarly, 78/286 (27.27\%) LVX MXF $^{\mathrm{R}}$ MTB strains were identified with missense mutations in $g y r B$ gene in which 71 strains harbored $g y r B$ mutations along with the mutations in gyrA gene, whereas $7 \mathrm{LVX}^{\mathrm{R}} \mathrm{MXF}^{\mathrm{R}}$ strains possessed D461H, N499S, T500N, G510A and G512R mutations only in $\operatorname{gr} B$ gene with $\operatorname{gr} A^{\mathrm{wt}}$. Total 7 novel mutations were detected in $\operatorname{gyr} B$ gene in this study. In addition, with other novel non-synonymous mutations in gyrB, the new mutations $\mathrm{D} 461 \mathrm{H}$ and $\mathrm{N} 499 \mathrm{~S}$ were found almost at the QRDR of gyrB which seems to be a very interesting phenomena of resistance in $\mathrm{LVX}^{\mathrm{R}} \mathrm{MXF}^{\mathrm{R}} \mathrm{MTB}$ strains. gyrB mainly carried missense mutations at codons D79N, T500A, G510A and G512R in $\mathrm{LVX}^{\mathrm{R}} \mathrm{MXF}^{\mathrm{R}}$ strains.

Among the 17/400 $\mathrm{LVX}^{\mathrm{R}} \mathrm{MXF}^{\mathrm{S}}$ strains, 10/17 (58.82\%) strains detected with multiple mutations in- and outside the QRDR of gyrA while 7/17 (41.17\%) strains had only a novel mutation at L105R in QRDR. Besides, A204V+D94G mutations frequently existed in gyr $A$ in this pattern of resistant strains. Likewise, 6/17 $\mathrm{LVX}^{\mathrm{R}} \mathrm{MXF}^{\mathrm{S}}$ gyr $A$ mutations containing strains were also noticed with a novel mutation R446L accompanied by mutations at A504T and G510A in gyrB gene.

Of the 6/400 $\mathrm{LVX}^{\mathrm{S}} \mathrm{MXF}^{\mathrm{R}}$ strains, gyr $A$ carried only novel mutations at D151T and V165A while gyrB showed G512R, D515G and R631S mutations in 7/400 $\mathrm{LVX}^{\mathrm{S}} \mathrm{MXF}^{\mathrm{R}}$ strains. Notably, in the current study, broad cross-resistance was noticed between LVX and MXF in 254/286 (88.81\%) $\mathrm{LVX}^{\mathrm{R}} \mathrm{MXF}^{\mathrm{R}}$ MTB strains by single or multiple mutations in gyr $A$ and/or gyrB gene(s). The non-synonymous mutations in gyrB conferred cross-resistance between LVX and MXF alone or in a combination of gyr $A$ gene, it enlightened the role of $\operatorname{gyr} B$ in development of resistance to FQ.

The $\mathrm{LVX}^{\mathrm{S}} \mathrm{MXF}^{\mathrm{S}}$ strains had $\operatorname{gyr} B^{\mathrm{wt}}$ or synonymous mutations (T37T, L568L), while gyrA was found with non-synonymous mutation (E21Q, S95T and G668D) in all strains. Interestingly, overall 18 novel mutations were detected in both genes. Two hundred and fortyfour out of two hundred seventy-seven $(88.08 \%)$ and $59 / 91(64.83 \%)$ resistant strains harbored mutations only in the QRDRs of gyrA and gyrB respectively. Whereas, 33 strains carried gyrA and 38 strains carried gyrB mutations outside the QRDRs among all FQ-resistant isolates in this study. Compared to the gyrA gene, gyrB had $(\leq 60 \%)$ less mutations which strengthen the discernment about gyr $A$ to account as a potential target of FQ in MTB. However, the most interestingly, 25/286 $\mathrm{LVX}^{\mathrm{R}} \mathrm{MXF}^{\mathrm{R}}$ MTB strains showed mutations at positions (E21Q, S95T and G668D) in the $\operatorname{gyr} A$ which were not associated with resistance. The same mutations were also present in all susceptible strains that indicates the existence of unknown resistance mechanisms in $\mathrm{FQ}^{\mathrm{R}} \mathrm{MTB}$ strains.

\section{Association of the Beijing and non-Beijing genotypes with gyrA and gyrB mutations}

The distribution of different mutation types in gyr $A$ and gyrB genes between Beijing and non-Beijing genotypes of MTB isolates were summarized in Table 2. Total 317/400 (79.25\%) MTB isolates belonged to the Beijing genotype, while $83 / 400(20.75 \%)$ were from non-Beijing genotypes. Among the total Beijing genotype strains, 225/317 (70.97\%) isolates harbored mutations in gyrA. Fifty-two out of eighty-three $(62.65 \%)$ non-Beijing genotypes also carried mutations in gyrA. The calculated $\mathrm{P}$ value was 0.14 for total gyrA mutations. The three mutations (E21Q, S95T and G668D) in gyrA were found in all MTB isolates of Beijing and non-Beijing genotypes. Moreover, 75/317 (23.65\%) MTB isolates with gyrB mutations belonged to Beijing genotype whereas $16 / 83$ (19.27\%) were from non-Beijing genotype. The $\mathrm{P}$ value was 0.39 for total gyrB mutations. Statistical analysis revealed no significant difference in prevalence of gyrA and $g y r B$ mutations between Beijing and non-Beijing genotypes. In the same way, there was also no significant difference was found in the distribution of the mutant gyrA and gyrB types between Beijing and nonBeijing genotypes. 
Table 2 Sequencing outcome of gyrA and gyrB genes versus Beijing and non-Beijing genotypes in MTB strains

\begin{tabular}{|c|c|c|c|c|c|c|c|c|}
\hline \multirow{2}{*}{ Genes } & \multicolumn{2}{|c|}{ Resistance pattern } & \multicolumn{2}{|c|}{ Mutations } & \multirow{2}{*}{ No. of strains } & \multicolumn{2}{|c|}{ No. of isolates with different genotypes $(n=400)$} & \multirow{2}{*}{$P$ value } \\
\hline & LVX & MXF & Nucleotide change & Amino acid & & Beijing $(n=317)$ & Non-Beijing $(n=83)$ & \\
\hline \multirow[t]{16}{*}{ gyrA } & $\mathrm{R}$ & $\mathrm{R}$ & C269T & A90V & 59 & 51 & 8 & 0.14 \\
\hline & & & G280T & D94Y & 14 & 10 & 4 & 0.46 \\
\hline & & & G280C & $\mathrm{D} 94 \mathrm{H}$ & 19 & 14 & 5 & 0.54 \\
\hline & & & A281C & D94A & 30 & 25 & 5 & 0.56 \\
\hline & & & C367T, G280A & $\mathrm{P}^{2} 3 \mathrm{~S}^{\#}, \mathrm{D} 94 \mathrm{~N}$ & 2 & 2 & 0 & 0.85 \\
\hline & & & G376A & A126E $E^{\#}$ & 4 & 4 & 0 & 0.55 \\
\hline & & & T380A & M127K & 2 & 2 & 0 & 0.85 \\
\hline & $\mathrm{R}$ & $S$ & G63T, T271C & E21H", S91P & 3 & 2 & 1 & 0.59 \\
\hline & & & T314G & $\mathrm{L}^{105 \mathrm{R}^{\#}}$ & 7 & 5 & 2 & 0.60 \\
\hline & & & C611T, A281G & A204V", D94G & 5 & 3 & 2 & 0.30 \\
\hline & & & G2426C, G280C & $\mathrm{G} 89 \mathrm{~A}^{\#}, \mathrm{D} 94 \mathrm{H}$ & 2 & 2 & 0 & 0.85 \\
\hline & $S$ & $\mathrm{R}$ & G451T & D151T" & 3 & 2 & 1 & 0.59 \\
\hline & & & T494C & V165A" & 3 & 3 & 0 & 0.68 \\
\hline & $\mathrm{S}$ & $\mathrm{S}$ & G66A & Q22Q & 1 & 1 & 0 & 0.88 \\
\hline & & & G591A & L197L & 1 & 1 & 0 & 0.88 \\
\hline & & & C2268T & D756D & 1 & 0 & 1 & 0.13 \\
\hline Total & & & & & & 225 (70.97) & 52 (62.65) & 0.14 \\
\hline \multirow{11}{*}{ gyrB } & & & A1498G & T500A & 10 & 9 & 1 & 0.41 \\
\hline & & & C1499A & $\mathrm{T} 500 \mathrm{~N}^{\star \star \phi}$ & 9 & 7 & 2 & 0.91 \\
\hline & & & G1529C & $\mathrm{G} 10 \mathrm{~A}^{* \star \phi}$ & 12 & 10 & 2 & 0.72 \\
\hline & & & G1534A & G512R ${ }^{\star \star \phi}$ & 13 & 11 & 2 & 0.62 \\
\hline & & & G1559C & G520A & 2 & 2 & 0 & 0.85 \\
\hline & $\mathrm{R}$ & $\mathrm{S}$ & G1337T, G1529C & $\mathrm{R}_{446 \mathrm{~L}^{\#}, \mathrm{G} 510 \mathrm{~A}}$ & 3 & 3 & 0 & 0.68 \\
\hline & & & G1510A & A504T & 3 & 3 & 0 & 0.68 \\
\hline & $\mathrm{S}$ & $\mathrm{R}$ & A1544G & D515G & 4 & 4 & 0 & 0.55 \\
\hline & & & C1891A & $\mathrm{R} 631 \mathrm{~S}^{\#}, \mathrm{G} 512 \mathrm{R}$ & 3 & 2 & 1 & 0.59 \\
\hline & $\mathrm{S}$ & S & C111T & T37T & 8 & 5 & 3 & 0.25 \\
\hline & & & G1704A & L568L & 4 & 3 & 1 & 0.83 \\
\hline Total & & & & & Total & 75 (23.65) & $16(19.27)$ & 0.39 \\
\hline
\end{tabular}

25 LVX $^{\mathrm{R}}$ MXF ${ }^{\mathrm{R}}$ MTB strains had (E21Q, S95T and G668D) mutations in the gyrA alike all susceptible strains, which are natural polymorphisms, not associated with resistance. These mutations are excluded from the table. Only non-synonymous mutations were included during analysis. The $\left.{ }^{* *}\right)$ and $\left(^{\phi}\right)$ mutations in gyrB were also present in gyrA ${ }^{w t}$ resistant strains. $\mathrm{P}<0.05$. ${ }^{\#}$, indicates the novel mutations in this study. LVX, Levofloxacin. MXF, Moxifloxacin. 


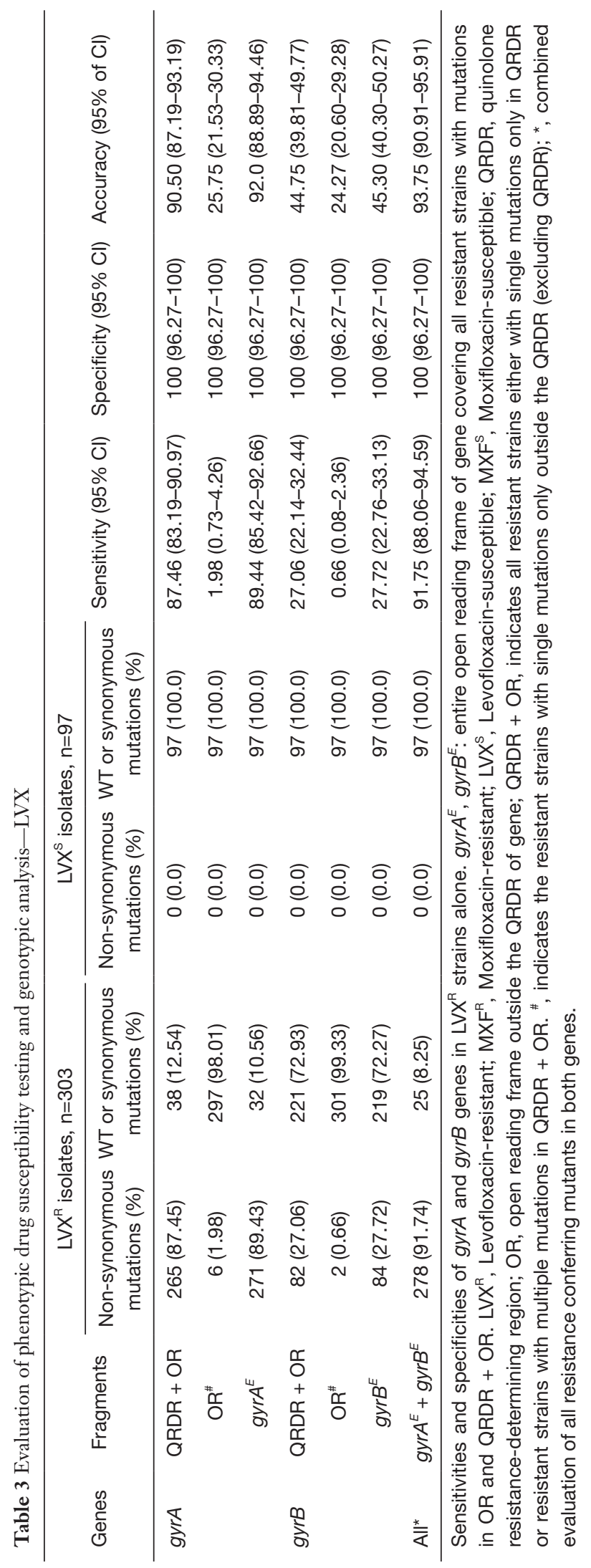

\section{Evaluation of $L V X^{R}$ and $M X F^{R} T B$ by DNA sequencing}

Phenotypic diagnostic veracity was compared with molecular diagnosis. Considering the phenotypic data as a reference, detection of mutation in the QRDR of $\operatorname{gyr} A$ for $\mathrm{LVX}^{\mathrm{R}}$ exhibited the sensitivity $87.46 \%$ and specificity $100 \%$, however when the entire gene is evaluated the sensitivity increased up to $89.44 \%$ while the specificity remained unchanged. The sensitivity of QRDR mutations in $g y r B$ was $27.06 \%$ while the entire gyr $B$ gene showed slightly increased sensitivity of $27.72 \%$ with the same specificity $100 \%$. When both genes were evaluated together, the sensitivity and accuracy increased up to $91.75 \%$ and $93.75 \%$ respectively (Table 3). The sensitivity of gyrA QRDR was $84.93 \%$ in $\mathrm{MXF}^{\mathrm{R}}$ strains but the entire gyrA gene showed the increased sensitivity of $89.04 \%$ with consistent specificity of $100 \%$. The sensitivity of gyrB QRDR in $\mathrm{MXF}^{\mathrm{R}}$ strains was $28.42 \%$ but it was increased to $29.11 \%$ in entire $g y r B$ gene. The combined evaluation of $g y r A$ and $g y r B$ enhanced the sensitivity to $91.44 \%$ with $100 \%$ specificity (Table 4 ). Detection of mutations in gyrA and gyrB to identify crossresistance in $\mathrm{LVX}^{\mathrm{R}} M \mathrm{MXF}^{\mathrm{R}}$ strains revealed a total sensitivity of $91.26 \%$ and a specificity of $100 \%$ (Table 5).

\section{Discussion}

FQ have been extensively used in China for more than a decade not only for treatment of MDR-TB but also for numerous bacterial infections. The exploitation of FQ drastically increased the number of FQ-resistant TB patients in China (5). In conformity with this theory, our data revealed a very high prevalence of FQ resistance particularly in southern China. To the best of our knowledge, this study comprises the highest collection of M/pre-X/X-DR MTB clinical isolates 321/400 (80.25\%) from southern China. A total of 303/400 (75.75\%) LVX ${ }^{\mathrm{R}}$ and 292/400 (73.00\%) MXF ${ }^{\mathrm{R}}$ MTB isolates were identified in this study which were considerably higher than the range of $50-70 \%$, obtained from the studies conducted in Taiwan (2), Morocco (14), China (36), Korea (37) and Belgium (38).

The high prevalence of $\mathrm{LVX}^{\mathrm{R}}$ and $\mathrm{MXF}^{\mathrm{R}}$ in pre-XDR and XDR $M$. tuberculosis isolates strongly underscores the need for more accurate susceptibility testing method to provide the guidance for appropriate MDR-TB regimens (39). DNA gyrase mutations were found to be associated with resistance or cross-resistance to the new generation of quinolones. The prevalence of gyrA mutations in quinoloneresistant clinical isolates varies across the countries, ranging 

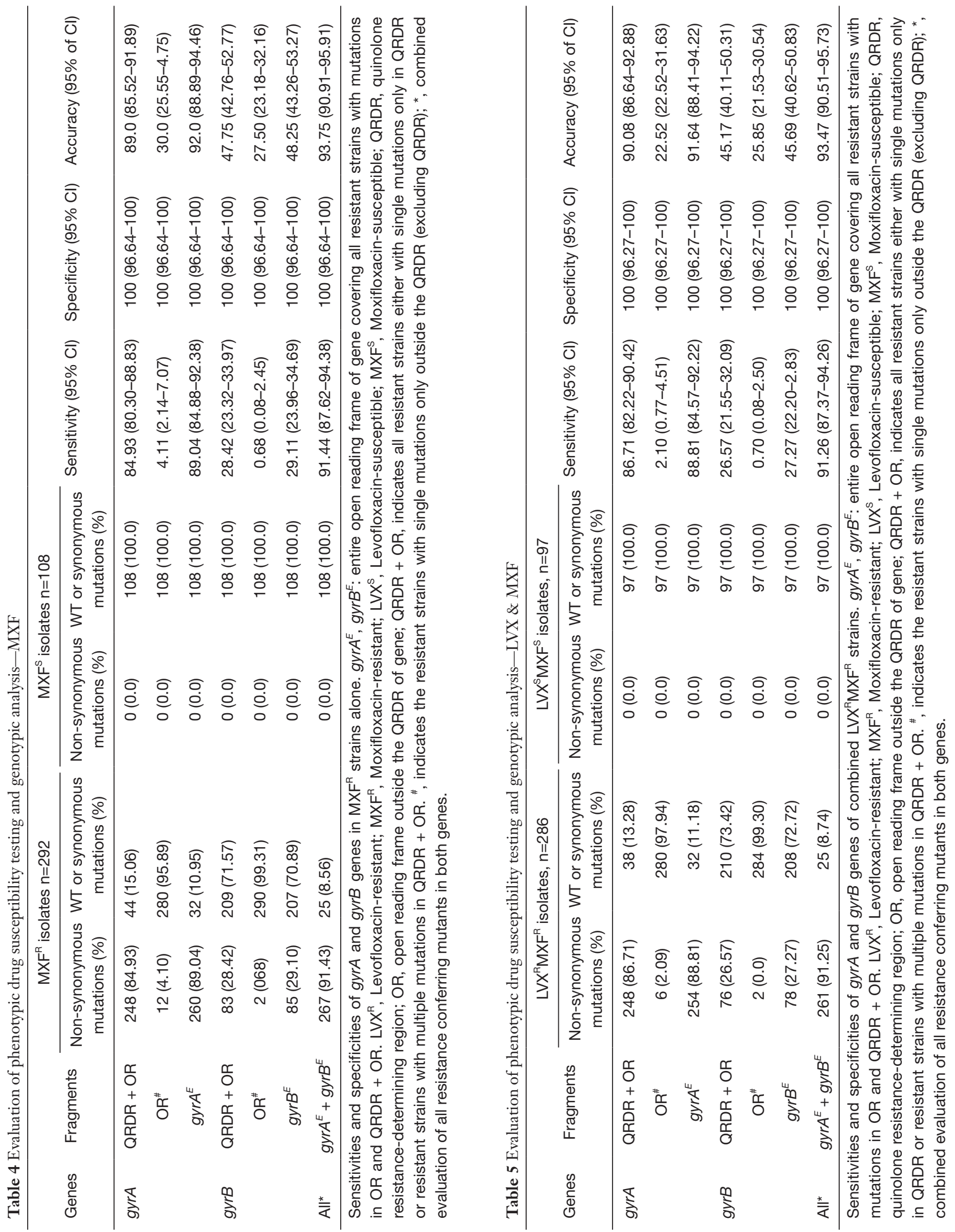
from $50 \%$ to $90 \%(5,40-43)$. In this study, we found $~ 90 \%$ of prevalence of gyrA mutations among the (271/303) $\mathrm{LVX}^{\mathrm{R}}$ and (260/292) $\mathrm{MXF}^{\mathrm{R}}$ strains which is noticeably higher than the studies from Beijing (68\%), Shanghai (76\%), Russia $(83 \%)$, Taiwan $(50 \%)$, Tunisia $(50 \%)$ and New York $(67 \%)$ $(40,41,44-47)$.

In our study, 244/278 (87.76\%) resistant strains had mutations in QRDR of gyrA with the most frequent mutations occurring at A90V, D94N, D94G and D94A. The mutations at D94 (D94G, D94A, D94N, D94H, D94Y) were the most detected mutations in QRDR. The D94 residue, that uphold the watermagnesium ion bridge with a conserved $\mathrm{C} 3 / \mathrm{C} 4$ keto acid moiety of quinolones, plays a significant role to stabilize the quinolone molecule in the $\mathrm{QBP}$, an amino acid substitution at 94 position will aggrandize the deleterious effect of the binding between quinolones and DNA gyrase $(48,49)$. D94 mutations were found to be associated with higher level of phenotypic resistance, as it might be the reason of positive epistasis between D94 mutations and the mutations eliciting rifampinresistance to develop MDR-TB to XDR-TB $(3,24,42,50)$. In addition, the mutations in $g y r A$ conferred resistance to $254 / 286(88.81 \%) \mathrm{LVX}^{\mathrm{R}} \mathrm{MXF}^{\mathrm{R}}, 17 \mathrm{LVX}^{\mathrm{R}} \mathrm{MXF}^{\mathrm{S}}$ and 6 $\mathrm{LVX}^{\mathrm{S}} \mathrm{MXF}^{\mathrm{R}} \mathrm{MTB}$ strains which indicates the resilient behavior of gyrA mutations in independent or crossresistance between LVX and MXF.

Several studies have demonstrated that less number of mutations are found in $\operatorname{gyr} B$ gene in FQ-resistant MTB strains. We found $91 \mathrm{LVX}^{\mathrm{R}}$ and/or $\mathrm{MXF}^{\mathrm{R}} \mathrm{MTB}$ clinical strains with missense mutations in $\operatorname{gr} B$ and $84 / 91(92.30 \%)$ of them also had mutations in $\operatorname{gyr} A$ gene. However, it is interesting to note that $7 \mathrm{LVX}^{\mathrm{R}} \mathrm{MXF}^{\mathrm{R}}$ MTB strains were detected with D461H, N499S, T500N, G510A and G512R mutations in gyrB gene only without any mutation in gyrA. Two of them $(\mathrm{D} 461 \mathrm{H}$ and N499S) are newly identified in this study. In agreement with the previous studies, T500A, G510A and G512R mutations were at high-level of mutation rate in $\operatorname{gyr} B$ of LVX $^{\mathrm{R}} \mathrm{MXF}^{\mathrm{R}}$ MTB strain (2,5). Furthermore, 78/91 $(85.71 \%)$ strains with $\operatorname{gyr} B$ mutations showed resistance to LVX and MXF similar to earlier reports of crossresistance between older generation of FQ in MTB $(3,4,14,51)$. The gyrB mutation rate was $\leq 30 \%$ in $(84 / 303)$ $\mathrm{LVX}^{\mathrm{R}}$ and $(85 / 292) \mathrm{MXF}^{\mathrm{R}}$ strains in our study which was markedly higher than previously published studies from Russia $12.5 \%$ (40), Shanghai $15.5 \%$ (47) and other parts of China $5.0 \%$ (33).
Based on the present data, non-synonymous novel nucleotide substitutions were also detected outside the QRDR(s) of gyrA (A126E, M127K, D151T, V165A) and $\operatorname{gyr} B(\mathrm{D} 461 \mathrm{H})$. These novel nucleotide substitutions were found without commonly existed mutations in QRDR(s) which showed their possible involvement in the development of LVX and/or MXF resistance. The repetition of phenotypic and genotypic testing methods in two independent experiments produced the same results in these strains. However, further molecular study will provide the new insight about the role of these novel non-synonymous nucleotide substitutions.

Moreover, the mutations in the QRDR(s) of gyrA and $\operatorname{gyr} B$ genes are widely accepted as the key reason for FQ resistance, so, we also identified the novel mutations (L105R) in QRDR(s) of gyrA and (N499S, G520A) in $g y r B$ for the first time. On the other hand, alike previous studies no resistance conferring non-synonymous mutations were detected in gyrB gene in $\mathrm{LVX}^{\mathrm{S}} \mathrm{MXF}^{\mathrm{S}}$ strains (52). However, in these strains gyr $A$ gene showed non-synonymous mutations (E21Q, S95T and G668D), but it has been proved that these non-synonymous mutations have no association with FQ resistance and thus considered as nonfunctional polymorphisms (52).

Generally, MTBDRsl (Hain Lifescience, Nehren, Germany) assay, identifies only the most common mutations found in the QRDR of gyrA $(16,17)$. Whereas in our study, compared with the phenotypic data, the sensitivities for identifying $\mathrm{LVX}^{\mathrm{R}}$ or $M \mathrm{MXF}^{\mathrm{R}}$ strains were detected by genotypic analysis of entire gyr $A$ and $\operatorname{gyr} B$ genes. The combined evaluation of novel and commonly existed resistance conferring mutations in entire open reading frames of $g y r A$ and $g y r B$ genes rather than sequencing only QRDR(s) improved the sensitivities up to $91.75 \%$ and $91.44 \%$ respectively with the $100 \%$ specificity which were higher than the sensitivities measured in previous studies from Taiwan: $67.7 \%$ (2), Vietnam: 75.6\% (20), France: 87\% (18) and Beijing: 89.7\% (17). The cross-resistance between LVX and MXF demonstrated the sensitivity of $91.26 \%$ and specificity of $100 \%$. This study shows the $\geq 90 \%$ concordance between phenotypic and genotypic diagnosis. Our findings enlighten that inclusion of entire open reading frames of gyrA and $g y r B$ in rapid molecular susceptibility testing will facilitate acquiring more accurate and dependable data of FQ resistance in MDR-TB. Total 18 novel non-synonymous mutations $(11$ gyr $A+7 \operatorname{gyr} B)$ are identified in our study which have never been reported in earlier studies. 
Most importantly, $25 \mathrm{LVX}^{\mathrm{R}} \mathrm{MXF}^{\mathrm{R}}$ strains were identified with (E21Q, S95T and G668D) mutations in gyr A accompanied by $g y r B^{\mathrm{wt}}$. These mutations were recognized as natural polymorphisms, not related with FQ resistance (52) which indicates the existence of unknown resistance mechanisms. However, alternative resistance mechanisms including an active drug efflux pump and reduced cell wall permeability to the drug to confer FQ resistance were suggested in previous studies but further molecular analysis will explore the new insights in FQ resistant MTB clinical strains $(2,5)$.

Moreover, in prior studies from Russia, Taiwan, Tunisia and Vietnam, Beijing genotype was considerably found to be associated with FQ resistance $(12,40,45)$. On the contrary, in our large number of MTB clinical isolates from southern China, no statistically significant prevalence of FQ resistance and mutant types were observed specifically bound with Beijing genotype, our findings are in line with previously published studies form China $(5,33)$. The mutations in gyr $A$ and $\operatorname{gyr} B$ were concomitantly distributed in Beijing and non-Beijing genotypes. The geographical discrepancy in prevalence of FQ resistant strains might be one of the promising reasons of varied profiles of mutant types and the extensive use of FQ for patients having bacterial infections might be another contributing factor for high emergence of drug resistance among MTB strains from different regions (23).

\section{Conclusions}

In conclusion, this is the first report which highlights the rising prevalence of independent and cross-resistance between LVX and MXF, commonly used for treatment of MDR-TB in southern China that is relatively higher than other countries. Genotypic analysis revealed the 18 novel mutations in addition to the highest prevalence rate of A90V, D94N, D94G and D94A mutations in gyrA as well as T500A, G510A and G512R mutations in gyrB in LVX $^{\mathrm{R}} \mathrm{MXF}^{\mathrm{R}}$ MTB strains. Our findings also demonstrated that mutations in QRDR(s) of gyrase genes are the most predominant mechanism accounting for $L V X^{R}$ and $M X F^{R}$ TB. However, the newly identified mutations in- and outside the QRDR of $\operatorname{gr} A$ (L105R, A126E, M127K, D151T, V165A) and gyrB (D461H, N499S, G520A) increased the sensitivity and the consistency of molecular diagnostic tests for rapid and more accurate diagnosis of FQ resistant strains. Mutations located in QRDR(s) were simultaneously associated with Beijing and non-Beijing genotypes. Most importantly, $25 \mathrm{LVX}^{\mathrm{R}} \mathrm{MXF}^{\mathrm{R}} \mathrm{MTB}$ strains without any known resistance-conferring mutations in gyrA and $\operatorname{gyr} B$ genes indicate the existence of unknown resistance mechanism which should be comprehensively investigated in forthcoming studies to explore the new potential target of FQ in MTB.

\section{Acknowledgments}

We thank the UCAS PhD Fellowship Program (to HMA Hameed) and CAS-TWAS President's PhD Fellowship Program (to MM Islam and C Chhotaray) for international students.

Funding: This work was supported by the National Natural Science Foundation of China (81572037, 81973372, 21920102003), the National Mega-project of China for Main Infectious Diseases (2017ZX10302301-003-002), the PhD. Start-up Fund of the Natural Science Foundation of Guangdong Province, China (2016A030310123), Science and Technology Department of Guangdong Province (2017A020212004, 2019B110233003).

\section{Footnote}

Conflicts of Interest: $\mathrm{T}$ Zhang received Science and Technology Innovation Leader of Guangdong Province (2016TX03R095). The other authors have no conflicts of interest to declare.

Ethical Statement: The authors are accountable for all aspects of the work in ensuring that questions related to the accuracy or integrity of any part of the work are appropriately investigated and resolved. The current study was conducted in accordance with WHO guidelines and approved by the Ethics Committee of Guangzhou Chest Hospital (GZXK-2016-015).

\section{References}

1. WHO. Global tuberculosis report 2018. Geneva, Switzerland: World Health Organization; 2018. WHO/ CDS/TB/2018.20.

2. Chien JY, Chiu WY, Chien ST, et al. Mutations in gyrA and gyrB among fluoroquinolone- and multidrug-resistant Mycobacterium tuberculosis isolates. Antimicrob Agents Chemother 2016;60:2090-6.

3. Farhat MR, Jacobson KR, Franke MF, et al. Gyrase mutations are associated with variable levels of 
fluoroquinolone resistance in Mycobacterium tuberculosis. J Clin Microbiol 2016;54:727-33.

4. Nosova EY, Bukatina AA, Isaeva YD, et al. Analysis of mutations in the gyrA and gyrB genes and their association with the resistance of Mycobacterium tuberculosis to levofloxacin, moxifloxacin and gatifloxacin. J Med Microbiol 2013;62:108-13.

5. Zhang Z, Lu J, Wang Y, et al. Prevalence and molecular characterization of fluoroquinolone-resistant Mycobacterium tuberculosis isolates in China. Antimicrob Agents Chemother 2014;58:364-9.

6. Ma Y, Pang Y, Du J, et al. Clinical outcomes for multiand extensively drug resistant tuberculosis patients with adjunctive resectional lung surgery in Beijing, China. J Thorac Dis 2017;9:841-5.

7. Lee EH, Yong SH, Leem AY, et al. Improved fluoroquinolone-resistant and extensively drug-resistant tuberculosis treatment outcomes. Open Forum Infect Dis 2019;6:ofz118.

8. Sun Z, Chao Y, Zhang X, et al. Characterization of extensively drug-resistant Mycobacterium tuberculosis clinical isolates in China. J Clin Microbiol 2008;46:4075-7.

9. Tang S, Zhang Q, Yu J, et al. Extensively drug-resistant tuberculosis, China. Emerg Infect Dis 2011;17:558.

10. Qi YC, Ma MJ, Li DJ, et al. Multidrug-resistant and extensively drug-resistant tuberculosis in multi-ethnic region, Xinjiang Uygur Autonomous Region, China. PLoS One 2012;7:e32103-e.

11. Li X, Wang H, Jing H, et al. Population-based surveillance of extensively drug-resistant tuberculosis in Shandong Province, China. Int J Tuberc Lung Dis 2012;16:612-4.

12. Maruri F, Sterling TR, Kaiga AW, et al. A systematic review of gyrase mutations associated with fluoroquinolone-resistant Mycobacterium tuberculosis and a proposed gyrase numbering system. J Antimicrob Chemother 2012;67:819-31.

13. Sun H, Zeng J, Li S, et al. Interaction between rpsL and gyrA mutations affects the fitness and dual resistance of Mycobacterium tuberculosis clinical isolates against streptomycin and fluoroquinolones. Infect Drug Resist 2018;11:431-40.

14. Chaoui I, Oudghiri A, El Mzibri M. Characterization of gyrA and gyrB mutations associated with fluoroquinolone resistance in Mycobacterium tuberculosis isolates from Morocco. J Glob Antimicrob Resist 2018;12:171-4.

15. Deshmukh SR, Chunarkar P, Islam MA. Insilico analysis of gyrase subunits a and $\mathrm{b}$ in prokaryotes. Int $\mathrm{J}$ Pharm Pharm Sci 2013;5:339-45.
16. Gao Y, Zhang Z, Deng J, et al. Multi-center evaluation of Geno'Type MTBDRsl line probe assay for rapid detection of pre-XDR and XDR Mycobacterium tuberculosis in China. J Infect 2018;77:328-34.

17. Jian J, Yang X, Yang J, et al. Evaluation of the GenoType MTBDRplus and MTBDRsl for the detection of drugresistant Mycobacterium tuberculosis on isolates from Beijing, China. Infect Drug Resist 2018;11:1627-34.

18. Brossier F, Veziris N, Aubry A, et al. Detection by GenoType MTBDRsl test of complex mechanisms of resistance to second-line drugs and ethambutol in multidrug-resistant Mycobacterium tuberculosis complex isolates. J Clin Microbiol 2010;48:1683-9.

19. Hillemann D, Rüsch-Gerdes S, Richter E. Feasibility of the Geno'Type MTBDRsl assay for fluoroquinolone, amikacin-capreomycin, and ethambutol resistance testing of Mycobacterium tuberculosis strains and clinical specimens. J Clin Microbiol 2009;47:1767-72.

20. Kiet VS, Lan NTN, An DD, et al. Evaluation of the MTBDRsl test for detection of second-line-drug resistance in Mycobacterium tuberculosis. J Clin Microbiol 2010;48:2934-9.

21. Hameed HMA, Islam MM, Chhotaray C, et al. Molecular targets related drug resistance mechanisms in MDR, XDR-, and TDR-Mycobacterium tuberculosis strains. Front Cell Infect Microbiol 2018;8:114.

22. Islam MM, Hameed HMA, Mugweru J, et al. Drug resistance mechanisms and novel drug targets for tuberculosis therapy. J Genet Genomics 2017;44:21-37.

23. Avalos E, Catanzaro D, Catanzaro A, et al. Frequency and geographic distribution of gyrA and gyrB mutations associated with fluoroquinolone resistance in clinical Mycobacterium tuberculosis isolates: a systematic review. PLoS One 2015;10:e0120470-e.

24. Willby M, Sikes RD, Malik S, et al. Correlation between GyrA substitutions and ofloxacin, levofloxacin, and moxifloxacin cross-resistance in Mycobacterium tuberculosis. Antimicrob Agents Chemother 2015;59:5427-34.

25. Juarez-Eusebio DM, Munro-Rojas D, Muñiz-Salazar R, et al. Molecular characterization of multidrug-resistant Mycobacterium tuberculosis isolates from high prevalence tuberculosis states in Mexico. Infect Genet Evol 2017;55:384-91.

26. Kim CH, Woo H, Hyun IG, et al. A comparison between the efficiency of the Xpert MTB/RIF assay and nested PCR in identifying Mycobacterium tuberculosis during routine clinical practice. J Thorac 
Dis 2014;6:625-31.

27. Sibley CD, Peirano G, Church DL. Molecular methods for pathogen and microbial community detection and characterization: current and potential application in diagnostic microbiology. Infect Genet Evol 2012;12:505-21.

28. Pang Y, Dong H, Tan Y, et al. Rapid diagnosis of MDR and XDR tuberculosis with the MeltPro TB assay in China. Sci Rep 2016;6:25330.

29. Jo KW, Ji W, Hong Y, et al. The efficacy of rifabutin for rifabutin-susceptible, multidrug-resistant tuberculosis. Respir Med 2013;107:292-7.

30. Koh WJ, Kang YR, Jeon K, et al. Daily $300 \mathrm{mg}$ dose of linezolid for multidrug-resistant and extensively drugresistant tuberculosis: updated analysis of 51 patients. J Antimicrob Chemother 2012;67:1503-7.

31. WHO. Policy guidance on drug-susceptibility testing (DST) of second-line antituberculosis drugs. Geneva: world health organization, 2008. WHO/HTM/ TB/2008.392.

32. WHO. Technical manual for drug susceptibility testing of medicines used in the treatment of tuberculosis. 2018. WHO/CDS/TB/2018.24.

33. Zhao LL, Chen Y, Liu HC, et al. Molecular characterization of multidrug-resistant Mycobacterium tuberculosis isolates from China. Antimicrob Agents Chemother 2014;58:1997-2005.

34. Mustafa S, Javed H, Hashmi J, et al. Emergence of mixed infection of Beijing/Non-Beijing strains among multidrug resistant Mycobacterium tuberculosis in Pakistan. 3 Biotech 2016;6:108.

35. Xu J, Wang B, Hu M, et al. Primary Clofazimine and bedaquiline resistance among isolates from patients with multidrug-resistant tuberculosis. Antimicrob Agents Chemother 2017;61:e00239-17.

36. Islam MM, Tan Y, Hameed HMA, et al. Detection of novel mutations associated with independent resistance and cross-resistance to isoniazid and prothionamide in Mycobacterium tuberculosis clinical isolates. Clin Microbiol Infect 2019;25:1041.e1-7.

37. Jnawali HN, Hwang SC, Park YK, et al. Characterization of mutations in multi- and extensive drug resistance among strains of Mycobacterium tuberculosis clinical isolates in Republic of Korea. Diagn Microbiol Infect Dis 2013;76:187-96.

38. Von Groll A, Martin A, Jureen P, et al. Fluoroquinolone resistance in Mycobacterium tuberculosis and mutations in gyrA and gyrB. Antimicrob Agents Chemother
2009;53:4498-500.

39. Dookie N, Sturm AW, Moodley P. Moxifloxacin resistance in the F15/LAM4/KZN extensively drug-resistant strain of Mycobacterium tuberculosis. Infect Drug Resist 2014;7:223-8.

40. Mokrousov I, Otten T, Manicheva O, et al. Molecular characterization of Ofloxacin-resistant Mycobacterium tuberculosisstrains from Russia. Antimicrob Agents Chemother 2008;52:2937-9.

41. Rigouts L, Coeck N, Gumusboga M, et al. Specific gyrA gene mutations predict poor treatment outcome in MDRTB. J Antimicrob Chemother 2016;71:314-23.

42. Singh P, Jain A, Dixit P, et al. Prevalence of gyrA and $\mathrm{B}$ gene mutations in fluoroquinolone-resistant and -sensitive clinical isolates of Mycobacterium tuberculosis and their relationship with MIC of ofloxacin. J Antibiot 2015;68:63.

43. Sirous M, Khosravi AD, Tabandeh MR, et al. Molecular detection of rifampin, isoniazid, and ofloxacin resistance in Iranian isolates of Mycobacterium tuberculosis by high-resolution melting analysis. Infect Drug Resist 2018;11:1819-29.

44. Ginsburg AS, Sun R, Calamita H, et al. Emergence of fluoroquinolone resistance in Mycobacterium tuberculosisduring continuously dosed moxifloxacin monotherapy in a mouse model. Antimicrob Agents Chemother 2005;49:3977-9.

45. Huang TS, Kunin CM, Shin-Jung Lee S, et al. Trends in fluoroquinolone resistance of Mycobacterium tuberculosis complex in a Taiwanese medical centre: 1995-2003. J Antimicrob Chemother 2005;56:1058-62.

46. Sullivan EA, Palumbo L, Ebrahimzadeh A, et al. Emergence of fluoroquinolone-resistant tuberculosis in New York City. Lancet 1995;345:1148-50.

47. Zhu C, Zhang Y, Shen Y, et al. Molecular characterization of fluoroquinolone-resistant Mycobacterium tuberculosis clinical isolates from Shanghai, China. Diagn Microbiol Infect Dis 2012;73:260-3.

48. Aldred KJ, Blower TR, Kerns RJ, et al. Fluoroquinolone interactions with Mycobacterium tuberculosisgyrase: Enhancing drug activity against wild-type and resistant gyrase. Proc Natl Acad Sci U S A 2016;113:E839-46.

49. Blower TR, Williamson BH, Kerns RJ, et al. Crystal structure and stability of gyrase-fluoroquinolone cleaved complexes from Mycobacterium tuberculosis. Proc Natl Acad Sci U S A 2016;113:1706-13.

50. Borrell S, Teo Y, Giardina F, et al. Epistasis between antibiotic resistance mutations drives the evolution of 
extensively drug-resistant tuberculosis. Evol Med Public Health 2013;2013:65-74.

51. Pienaar E, Sarathy J, Prideaux B, et al. Comparing efficacies of moxifloxacin, levofloxacin and gatifloxacin in tuberculosis granulomas using a multi-scale systems pharmacology approach. PLoS Comput Biol
2017; 13:e1005650.

52. Lau RWT, Ho PL, Kao RYT, et al. Molecular characterization of fluoroquinolone resistance in Mycobacterium tuberculosis: functional analysis of gyrA mutation at position 74. Antimicrob Agents Chemother 2011;55:608-14.

Cite this article as: Hameed HMA, Tan Y, Islam MM, Guo L, Chhotaray C, Wang S, Liu Z, Gao Y, Tan S, Yew WW, Zhong N, Liu J, Zhang T. Phenotypic and genotypic characterization of levofloxacin- and moxifloxacin-resistant Mycobacterium tuberculosis clinical isolates in southern China. J Thorac Dis 2019;11(11):4613-4625. doi: 10.21037/jtd.2019.11.03 